\title{
Individual Differences in Second Language Learning: Introduction
}

\author{
Leah Roberts \\ University of York and Max Planck Institute for Psycholinguistics
}

\author{
Antje Meyer \\ Radboud University and Max Planck Institute for Psycholinguistics, Nijmegen
}

The topic of the workshop from which this volume comes, "Individual Differences in Second Language Learning," is timely and important for both practical and theoretical reasons. The practical reasons are obvious: While many people have some knowledge of a second or further language, there is enormous variability in how well they know these languages. Much of this variability is, of course, likely to be due to differences in the time spent studying or being immersed in the language, but even in similar learning environments learners differ greatly in how quickly they pick up a language and in their ultimate level of proficiency. For purposes of selecting and advising learners, and for identifying those who may require specific types of intervention, it would be extremely useful to be able to predict how quickly and how well an individual learner will acquire a new language and which type of instruction would suit them best. To offer such guidance we need suitable ways of assessing proficiency and we need tests that predict an individual's speed and learning success. During the workshop much of the discussion centered on these questions: how factors such as proficiency and language aptitude can be best defined and assessed; what assessable precursors of good second language learning might be; and how assessment tools can be developed, improved, and validated. These discussions are reflected in many of the articles in this volume (Dale, Harlaar, \& Plomin; Andringa, Olsthoorn, van Beuningen, Schoonen, \& Hulstijn; Sparks;

Correspondence concerning this article should be addressed to Leah Roberts, Centre for Language Learning Research, Department of Education, University of York, Heslington, York, YO10 5DD, UK. Internet: leah.roberts@york.ac.uk 
DeKeyser). With regard to precursors to successful language learning, one important topic of a number of articles is the relationship between skills in one's L1 and L2 learning ability. For instance, Sparks reports an impressive program of longitudinal studies, showing that individual differences in early L1 skills (e.g., in phonological awareness and word decoding) are related to differences in L2 learning ability. Also taking a longitudinal approach, Dale et al. discuss data from the Twins Early Development Study which show that the genetic factors that contribute to individual differences in L2 success may be in the main distinct from those that influence L1 achievement.

There are also important theoretical reasons for studying individual differences in second language learning and in psycholinguistics in general. Much of adult psycholinguistics has been oriented toward understanding the average speaker or listener. This is largely because of the affinity of psycholinguistics to general psychology, dealing with what is common to all people (as opposed to differential psychology, dealing with differences between people) and to linguistics, which is primarily concerned with the knowledge shared by all speakers of a language. Yet a comprehensive psychological theory of a skill or type of behavior should not only make predictions about average behavior, but also about individual differences. For instance, a theory that entails that working memory is required to learn grammatical rules predicts that differences in working memory capacity between learners should be associated with differences in their ability to learn grammatical rules. If this prediction is not borne out, the theory may need to be revised.

Thus, examining individual differences is an important way of testing and refining psychological theories. In fact, considering individual variation is often the best way of testing hypotheses. This is because it can be difficult to induce variation experimentally that exists naturally between people. This holds, for instance, for variables such as general intelligence or age. Their effects on language learning can only be assessed by comparing groups of learners differing in age or general intelligence or by examining how differences in age or intelligence within a group are related to learning outcomes. Correlational approaches - relating differences in predictor variables to differences in learning outcomes - are also an excellent way of studying how several variables jointly affect a target behavior. Many authors in this volume advocate the use of correlational approaches and experimental designs that allow for the modeling of continuous individual-level characteristics (e.g., proficiency), rather than forming post hoc groups of participants (DeKeyser; Roberts; Van Hell \& Tanner), and the articles by Andringa et al., Sebastián-Gallés and Díaz, and DeKeyser illustrate the benefits of the use of correlational approaches for 
second language acquisition research. For instance, Andringa et al. report that variation in both their native and non-native groups' listening comprehension ability was affected by linguistic knowledge. However, the authors' use of a multivariate design allowed for the assessment of variables in conjunction with each other, and they were also able to show that, for native speakers, differences in listening ability were also a function of processing speed, whereas, for the L2 learners, it was reasoning ability (IQ) that made a smaller, but still significant contribution.

The outcomes of such research can have broad theoretical consequences, especially when second language learning is compared to first language acquisition or when first and second language use in adult speakers are compared. Such comparisons can provide important clues about the way knowledge of the first language might support or hinder the acquisition of a second language. It can also illuminate how important general cognitive skills and abilities such as attention, inhibition and working memory, are in using a first or second language and whether the contributions of such general cognitive components to using language depend on the speakers' linguistic proficiency. Van Hell and Tanner discuss studies that investigate the relationship between L1 and L2 lexical processing. They demonstrate that with increased L2 proficiency one observes an influence on L1 processing and increased attentional and cognitive control as well as an increased ability to filter out irrelevant or inappropriate information. Similarly, such work can shed light on the architecture of the cognitive system generally, as shown in the work discussed by Sebastián-Gallés and Díaz on L1 and L2 speech perception. In other words, studying individual differences in second language learning can contribute to a better understanding of one of the key issues in current cognitive science, which is how general cognitive skills and domain-specific skills jointly determine behavior.

Currently, research aiming to develop and improve tools for the assessment of second language proficiency and research using individual differences to understand theoretical issues in language learning are distinct research fields. As Dale observes, questions of general mechanisms and questions of individual differences are different scientific research tasks and typically require different research methodologies. The articles in this volume clearly reflect this. The research goals range from assessing broadly defined constructs, especially language aptitude (e.g., Andringa et al.; Xiang, Dediu, Roberts, van Oort, Norris, \& Hagoort), to assessing very specific skills, such as the ability to learn novel vowel contrasts (Hanulíková, Dediu, Fang, Bašnaková, \& Huettig) and the assessment methods range from collecting large quantities of questionnaire data (Sparks) and teachers' ratings of ability over a number of years (Dale, Harlaar, \& 
Plomin) to tracking the learners' success in consonant discrimination tasks over several experimental sessions (Hanulíková et al.) to investigate the activation of language pathways in the brain (Xiang et al).

The division of labor between classroom-based and laboratory-based research is in part dictated by practical considerations; many state-of-the-art laboratory techniques cannot be exported to the classroom and used for testing large groups of learners. However, portable versions of many classic psycholinguistic tools have been developed (e.g., there are lexical decision apps for smartphones), and this development will undoubtedly continue. Therefore it will be increasingly possible to quickly assess learners on a variety of tasks and determine how well their performance in tasks measuring specific skills (e.g., speeded lexical access or syntactic parsing) correlates with broad assessments of their linguistic proficiency through language aptitude tests or teachers' ratings. This is an important development because it should lead to a better understanding of what laboratory tasks, questionnaires, and ratings actually measure. As DeKeyser notes, this in turn, will improve theories of second language learning and support the development of assessment instruments that are grounded in both educational practice and knowledge of the cognitive processes underlying language use.

In sum, the articles in this volume provide an overview of what we currently know about individual differences in second language learning and offer a wealth of ideas from researchers in different disciplines as to how to develop the field of individual differences in second language learning. This is fertile ground for new research that has the potential to impact language learning and general psychological theory, as well as educational practice. 